\title{
Die Verantwortung des Journalisten: Zum Problem der Pressefreiheit im Alltag des Lokal-Redakteurs aus christlicher Sicht
}

\author{
von K. Rüdiger Durth
}

\section{Festreden widerspricht man nicht}

Journalistische Verantwortung und Pressefreiheit markieren wechselseitig ein Problem unserer pluralistischen Gesellschaft, die zunehmend Medienpolitik als vorrangige Gemeinschaftsaufgabe erkennt. Jedoch ist die Gesellschaft aufgrund der ebenso unterschiedlichen wie gegensätzlichen Interessen und Ansprüche seitens der Verbände und Parteien offensichtlich nicht in der Lage, eine gemeinsame Problemlösung zu finden; diese wird durch wirtschaftliche Schwierigkeiten der Tagespresse noch zusätzlich erschwert.

Nach der Aufhebung von Presselizenzen und -zensur durch die alliierten Besatzungsmächte 1949 und durch den Schutz der Presse- und Meinungsfreiheit im Artikel 5 des Grundgesetzes wurde in der breiten Offentlichkeit der Bundesrepublik Deutschland die langentbehrte Pressefreiheit bald als eine Selbstverständlichkeit hingenommen. Dies änderte sich 1962, als auf dem Höhepunkt der "Spiegel-Krise" zehntausende meist junger Menschen für die Pressefreiheit auf die Straße gingen. In der Folge der Studentenrevolte 1968/69 kam es zu entgegengesetzten Aktionen, als von der Straße her versucht wurde, mit „Enteignet Springer"-Parolen und angezündeten Verlagswagen die Freiheit der Springer-Zeitungen einzuschränken.

Dies war freilich ein kurzes Wetterleuchten, das in das Gewitter einer umfangreichen Pressekonzentration überleitete ${ }^{1}$, die landesweit die Pressefreiheit zum politischen und gesellschaftlichen Thema machte. Wurde bislang Pressefreiheit in erster Linie verstanden als „Unabhängigkeit der Kommunikatoren vom staatlich-exekutiven Bereich bis zu dem Grade, daß Funktionen der öffentlichen Kritik und wirksamen Kontrolle der Herrschaftsschicht wie der Machtausübung ermöglicht werden “2, so ist nun die Frage der inneren Pressefreiheit und der Freiheit des Bürgers, sich durch verschiedene, miteinander konkurrierende Medien informieren zu können, in den Vordergrund getreten.

Diesen Umschwung im Verständnis von Pressefreiheit - was die Freiheit gegenüber dem Staat betrifft, zählt die deutsche Presse nach wie vor zu der freiesten in der Welt ${ }^{3}$ - verursachten aber auch folgende Faktoren:

Die Ideologisierung zahlreicher Journalisten in der Folge der (linken) Medienkritik die vermehrten Angriffe auf Rundfunk und Fernsehen seitens der Parteien bis hin zum Wort vom Westdeutschen Rundfunk als "Rotfunk" nalistischer Mitbestimmung in den Verlagen ${ }^{6}$ und vor allem das plötzlich erwachte Bewußtsein von der Bedeutung und den Gefahren der Massenkommunikationsmittel.

X. Rüdiger Durth ist Redakteur der „Kölnischen-Bonner Rundschau“. In Marburg und Bonn studierte er evangelische Theologie. 1973 wurde er zum Pastor der Evangelischen Kirche im Rheinland ordiniert. 
Die „Macher“ vieler Medien sind nun selbst in den Mittelpunkt öffentlichen Interesses gerückt.

Die eigentliche Auseinandersetzung freilich spielt sich (nicht nur erst) heute in den Redaktionen ab. Wer verantwortet - und darauf soll in diesem Beitrag der Schwerpunkt liegen - in den Redaktionen der Lokal- und Regionalzeitungen die alltägliche Pressefreiheit? Denn unter den deutschen Tageszeitungen verfügt diese Gruppe über die höchste Auflage ${ }^{7}$, arbeiten in diesen Redaktionen die meisten der über 20.000 hauptberuflichen Journalisten ${ }^{8}$. Im kommunalen Lebensraum findet nicht nur eine „entscheidende Prägung politischer Einstellungen“ statt ${ }^{9}$, sondern entbrennt auch der Interessenkampf am heftigsten, weil hier Handlungen und Ereignisse besonders namhaft, konkret und leicht überprüfbar werden ${ }^{10}$.

Die Lokalredakteure produzieren nicht nur „täglich zehnmal soviel Nachrichten und kommentierende Artikel wie die Kollegen in den politischen Ressorts" ${ }^{11}$, sondern sie sind in der Regel auch weniger gut in der Offentlichkeit angesehen ${ }^{12}$, oft finanziell schlechter gestellt ${ }^{13}$ und haben weniger Möglichkeiten zur Weiterbildung, übrigens eines der Probleme, die am wenigsten gelöst sind ${ }^{14}$.

Und nicht zuletzt vollzieht sich im lokalen Kommunikationsraum die engste Begegnung zwischen Kirche und Presse im Spannungsfeld von Anspruch und Widerspruch $^{15}$.

Sind sich alle in der Forderung nach Pressefreiheit einig, so trifft dies nicht mehr für die konkrete Verantwortung zu, mit der erst Pressefreiheit alltägliche Wirklichkeit wird. Denn Verantwortung wird subjektiv begründet und verteidigt. Die „Publizistischen Grundsätze" des Deurschen Presserates aus dem Jahre $1973^{16}$ sind so weit gefaßt, daß sie dem Journalisten großßen Spielraum, aber kaum Hilfe für die täglichen Entscheidungen geben. Auf der "Gegenseite", den Informanten einerseits und den Lesern andererseits, wird Verantwortung wiederum jeweils subjektiv als Forderung an den Journalisten erhoben, obgleich diese Forderung oft genug mit dem Anspruch der Objektivität zur besseren Durchsetzung eigener Interessen dient.

Im Hintergrund steht die Frage nach der Ethik des Journalisten, mit der er Pressefreiheit "praktiziert". In Festreden läßt sich die Ethik des Journalisten einleuchtend und breiten Konsens erzeugend trefflich formulieren. Doch den Festreden ist der appellative Charakter eigen, und die Redner bleiben meist die Antwort schuldig, wie denn nun die vielfältigen Imperative in die Wirklichkeit umgesetzt werden können. Anders gesagt: Die Frage ist bislang kaum beantwortet, wie der Journalist im Alltag mit "mannigfach großer - und meist kleiner Verantwortung ${ }^{{ }_{117}}$ fertig wird, nach welchen Kriterien er dieser Verantwortung gerecht zu werden versucht.

Festreden widerspricht meist niemand. Widerstand und Widerspruch werden sehr schnell spürbar, wenn das "Festgeredete" in den Alltag umgesetzt wird. Dann schließt sich keine Gruppe aus, auch die Kirche nicht, von der Michael Schmolke im Blick auf die katholische Kirche feststellt: „Die Kirche wird in Gegenwart und Zukunft weiterhin ihre Schwierigkeiten mit den Medien haben: Kirche und Publizistik das wird, solange wir Publizistik als ein liberales Prinzip verstehen und schätzen, immer ein gebrochenes Verhältnis bleiben. ${ }^{{ }{ }^{18}}$

Die Theorie von der Verantwortung hat nur dann einen Sinn, wenn sie im konkreten Alltag praktikabel ist. Dem Journalisten am Redaktionsschreibtisch geht es nicht anders als einem Menschen, der mit einem Tausendmarkschein eine Straßenbahn- 
fahrkarte lösen will. Es ist aussichtslos. Auch die tägliche Pressefreiheit wird stets mit kleinen Münzen bezahlt und nicht mit großen Scheinen.

Diese kleinen Münzen werden oft genug dem Journalisten vorenthalten oder - aus Unkenntnis über Arbeitsweise und -bedingungen seitens der Interessenvertreter streitig gemacht. Hier kann die Kirche eine wichtige Funktion erfüllen, die aber nur selten genutzt wird.

Vom katholischen „Welttag der sozialen Kommunikationsmittel“ beispielsweise erfahren die meisten Journalisten nichts, denn er spielt sich auf den Kanzeln ab (unter denen nur selten Journalisten sitzen), oder er wird mit theoretischen Aufsätzen in der Bistumspresse abgehandelt ${ }^{10}$.

Selbst dieser Tag also wird zum kirchlichen "Informationsgrab" ${ }^{20}$, obwohl er den verantwortlichen Priestern vor Ort eine gute Möglichkeit bieten würde, das Gespräch mit den Journalisten der Regional- und Lokalzeitungen zu suchen, sich über Massenmedien zu informieren und Denkanstöße für die Tagesarbeit zu vermitteln.

Die Kirche muß endlich begreifen, daß sie eine ganz konkrete Verantwortung für die Massenmedien und deren Mitarbeiter hat - freilich nicht im Sinn eines verdeckten oder offenen Machtanspruchs, sondern als Dienst an Menschen, die in der heutigen Gesellschaft eine besondere Verantwortung erfüllen. Robert Geisendörfer hat diese unmißverständlich deutlich gemacht: "Wenn Kommunikationspolitik weitgehend unter der Fragestellung, Was nützt es meiner Gruppe?‘ stattfindet, wenn das Allgemeinwohl keinen Anwalt mehr hat, dann muß die Kirche diesen leeren Fleck füllen." ${ }^{29}$

\section{Im Widerstreit der Interessen}

Die Zeitungsredaktion erhält, so definiert Manfred Rühl, „ihre besonderen Charakteristika erst durch die Be- und Verarbeitung von Informationen aus der Umwelt und durch die Bereitstellung der be- und verarbeiteten Information für die Umwelt. "22 Diese "Umwelt" ist im lokalen Kommunikationsraum nicht nur überschaubar (was von Vorteil ist), sondern auch vom hautnahen Interessenkampf und -ausgleich geprägt. Der plurale, weithin bereits pluralistische Kampf der verschiedenen Interessen wirkt sich besonders in einem Anspruchsdenken gegenüber der Zeitung aus.

Was im überregionalen Bereich für Schlagzeilen sorgt (Druck von Parteien oder Verbänden auf Massenmedien), aber meist ohne konkrete Auswirkungen bleibt ${ }^{23}$, bleibt im regionalen Bereich ohne Aufsehen. Der ausgeübte Druck ist subtiler, darum aber nicht weniger wirkungsvoll. Im Gegenteil.

Die Ursachen sind offenkundig: Die Lokalzeitung ist in weit höherem Maß auf jeden Abonnenten und Anzeigenkunden angewiesen als die überregionale Presse. Der Lokaljournalist ist den Interessengruppen besser bekannt als der anonyme Agenturjournalist oder Korrespondent. Dadurch wird der Druck auf den einzelnen leichter und zwar im Verhältnis zur Auflage der Zeitung. Je kleiner die Zeitung, desto größer die Erfolgsaussicht.

Der Entzug guter Informationsquellen im Rathaus beispielsweise kann für den Lokaljournalisten "tödlich" sein - im Gegensatz etwa zum Informationsentzug durch ein Ministerium für einen unbequemen Journalisten, der sich sofort zum Politikum hochspielen läßt und die jeweilige Opposition auf den Plan ruft ${ }^{24}$. 
Was für das Rathaus, die Parteien, die Gewerkschaften, die örtlichen Interessengruppen und Vereine gilt, gilt existentiell für die gesamte Zeitung im Blick auf die Anzeigenkunden, die verständlicherweise stets ein Interesse daran haben, auch im redaktionellen Teil berücksichtigt zu werden. Außerdem setzen sie stillschweigend voraus, daß sie nicht selbst zum Gegenstand kritischer Recherchen werden (etwa bei Bauplänen, die eine Änderung bestehender Bauverordnungen voraussetzen) und daß ihre allgemeinen wirtschaftlichen Interessen in der Berichterstattung gewahrt werden.

Die einzelnen Gruppen also betrachten die Zeitung als die „ihre“, um so ihren Interessen beim Leser (als Wähler, Mitglied, Kunden) zum Durchbruch zu verhelfen.

Der Leser wiederum will informiert werden, will Informationen über seinen engeren Lebensbereich erhalten, will wissen, was im Rathaus los ist, was er an Steuern zahlen muß, welches kulturelle Angebot zur Auswahl steht. Er verlangt Unterhaltung und Nachrichten über andere Menschen und zugleich auch ein Stück Kontrolle öffentlicher und institutioneller Gewalt. Er will informiert werden über seinen Verein, seine Kirche, seine Partei, die Schule seiner Kinder, über den Verkehrsunfall vor seiner Haustür.

Alle diese legitimen Ansprüche bedrängen die Redaktion, die nach ihrem Selbstverständnis keineswegs „zum Veröffentlichungsinstrument der Herrschenden und der Verwaltungen verkümmern" will ${ }^{25}$.

Die Redaktion wiederum stellt eine Gemeinschaft dar, die sowohl die internen als auch die von außen an sie herangetragenen Interessen ausgleichen muß. Sie arbeitet ständig gegen die Uhr, kämpft mit Platzmangel. Und doch muß sie dafür sorgen, ein möglichst umfassendes Bild über das aktuelle Geschehen des Tages zu vermitteln.

Die Lokalredaktionen wissen um die harte Kritik, die Kritiker an ihrer Arbeit üben: Die Lokalzeitung stellt einen "beschaulichen Schonraum" dar, die Obrigkeit spielt eine "dominierende Rolle" oder "das Auftreten der Honoratioren bildet den Anlaß der Berichterstattung " ${ }^{\text {"26 }}$. Aber wissen die Kritiker auch um die subtilen Sanktionen, die auf eine Nichtbeachtung eben der Honoratioren folgen (hier schließt sich die Ortskirche keinesfalls aus)?

Aufgrund ihrer schlechten personellen Besetzung sind die Lokalredaktionen auf zahlreiche Mitarbeiter angewiesen ${ }^{27}$, die weder journalistisch ausgebildet, noch oft zur kritischen Distanz fähig sind. Diesen Mitarbeitern fehlt es allzuoft an der notwendigen Allgemeinbildung, und außerdem sind sie in einem noch größeren Maß auf das Wohlwollen ihrer Informanten angewiesen als die Redakteure.

Und der Redakteur muß sich auf diese Mitarbeiter verlassen, weil es ihm in der Regel an der Zeit fehlt, die Manuskripte nachzurecherchieren, und er außerdem den Grundsatz zu beachten hat: Besser ein schlechter Bericht als gar keiner. Eine bittere, aber leider nur zu of notwendige Einsicht.

Ein weiterer Aspekt muß berücksichtigt werden: Rathäuser und Verbände haben längst die Bedeutung der lokalen Kommunikation erkannt und deshalb hauptamtliche Pressereferenten angestellt, die zum Teil eine vorzügliche Pressearbeit leisten. Aufgrund der guten Bezahlung können sich die Städte und Großgemeinden heute oft sehr qualifizierte Journalisten „leisten“, die nicht selten ihren Kollegen in den Redaktionen überlegen sind. Das führt u. a. dazu, daß die Redaktionen diese offi- 
ziellen Mitteilungen ohne Nachrecherche übernehmen. Und die Pressereferenten werden schließlich dafür bezahlt, die offizielle Rathauspolitik zu verkaufen ${ }^{28}$.

Diese Beispiele - in gedrängter Kürze und damit zwangsläufiger Verkürzung sollen den Hintergrund aufzeigen, auf dem von vielen Lokaljournalisten täglich gearbeitet wird, eine Arbeitsleistung verlangt wird, deren Bedeutung langsam ins öffentliche Bewußtsein dringt.

Angesichts dieser "materiellen" Schwierigkeiten (die um zahlreiche Beispiele ergänzt werden könnten, etwa die Tatsache, daß den meisten Lokalredaktionen ein gutgeführtes Archiv fehlt) und der großen Arbeitsintensität (die 40-Stunden-Woche steht für Journalisten nicht einmal auf dem Tarifpapier, und für einen Sonntagsdienst erhält der Redakteur nach geltendem Tarifrecht - immerhin lohnsteuerfrei ganze 25 DM) nimmt es kein Wunder, daß die Kritiker Anlaß zur Kritik finden. Verwundern müßte den Außenstehenden hingegen mehr die Tatsache, daß wir in der Bundesrepublik Deutschland iber eine sehr große Zahl ausgesprochen guter Lokalteile verfügen ${ }^{20}$.

Hinzukommt auch die Tatsache, daß viele qualifizierte Lokalredakteure versuchen, sehr schnell in ein anderes Ressort zu wechseln, weil der Lokalredakteur kaum Möglichkeiten der Spezialisierung hat, über geringes öffentliches Ansehen verfügt und of genug niedriger bezahlt wird als seine Kollegen in anderen Redaktionen.

\section{Die Lokalzeitung - ein Lazarett?}

Damit ist freilich noch nicht die Frage der Verantwortung des Journalisten beantwortet. Im Widerstreit der Interessen können nämlich höchstens die Bedingungen aufgezeigt werden, unter denen es schwerfällt, verantwortungsvoll zu handeln, können die Gründe sichtbar gemacht werden, die mangelnde Sorgfalt in der Recherche (um ein Beispiel zu nennen) entschuldigen, nicht aber rechtfertigen.

Die Veranwortlichkeit, zu der ein Journalist (und keineswegs nur der lokale) fähig ist, wird durch folgende Faktoren entscheidend mitbestimmt: Schul- und Ausbildung, selbstgesteckte Berufsauffassung, weltanschauliche Verankerung, Arbeitsmarktlage. Diese Faktoren lassen sich nicht scharf voneinander trennen, sondern bilden meist eine untrennbare Einheit. Um der Deutlichkeit willen soll eine Systematisierung versucht werden, die in der Praxis jedoch so klar nicht zutage tritt.

Der Arbeitsmarkt spielt heute für den Journalisten eine entscheidende Rolle. Er sieht düster aus ${ }^{30}$ und wird sich voraussichtlich nicht schnell ändern. Weniger Zeitungen auf dem Markt führen automatisch zu weniger Arbeitsplätzen. Die angespannte Wirtschaftslage zahlreicher Verlage zwingt zu Einsparungen im Personalsektor, der an den Kosten einer Zeitung einen hohen Anteil hat ${ }^{31}$. Gleichzeitig aber drängen viele junge Menschen in den journalistischen Beruf, sowohl Abiturienten als auch Jungakademiker, nicht zuletzt aber auch weiterhin viele Studenten, die ihr Studium abbrechen und im Journalismus einen Beruf sehen, den man schnell erlernen $k^{3 a n n}{ }^{32}$.

Diese Situation erzeugt Angst und eine schleichende Anpassung, in der jeder Konflikt vermieden wird. Die Sorge um den Arbeitsplatz rangiert an erster Stelle und nicht nur bei den älteren Redakteuren. Diese Entwicklung wird zwar nach außen bestritten - und wird keineswegs von Verlagsleitungen oder Chefredaktionen für 
gut befunden -, muß aber berücksichtigt werden. Die Folgen einer solchen Haltung, sollte sie sich noch weiter ausbreiten, sind für eine unabhängige und verantwortungsbewußte Berichterstattung verheerend.

Nebenbei sei die Frage gestellt, ob im Interesse der Unabhängigkeit des Journalisten nicht ein Zweikampf wünschenswert wäre, doch dürfte dies mit Ausnahme der Akademiker unter den Redakteuren praktisch kaum durchführbar sein.

Viele junge Menschen, die Journalist werden wollen, sind davon überzeugt, mit Hilfe der Zeitung „die Welt zu verändern“. Diese Erwartung wird naturgemäß sehr schnell enttäuscht ${ }^{33}$, dennoch bleibt dieses Motiv für viele maßgebend. Und man wird es nicht von vornherein negativ beurteilen können. Die oft befürchtete „Linksunterwanderung" der Medien hat inzwischen viel von ihrem Schrecken eingebüßt. Nicht weniger gefährlich ist die festzustellende Anpassung junger Menschen an vorhandene, oft eingefahrene Strukturen, um die Möglichkeit eines Volontariats bzw. einer journalistischen Karriere nicht zu gefährden. Die ethische Begründung journalistischen Handelns ist bei vielen jungen Journalisten recht verschwommen ${ }^{34}$.

Unsicherheit besteht auch darüber, was eigentlich als Voraussetzung für den journalistischen Beruf verlangt werden mu133. Das Wort von der "guten Allgemeinbildung“ stellt letztlich nicht mehr als einen alles und nichts sagenden Allgemeinplatz dar. Vom Lokaljournalisten müssen aber gründliche Kenntnis der Zeitgeschichte, der Gemeindeordnung, des allgemeinen Rechts, die Grundzüge der Kommunikationswissenschaft und der Soziologie als Eingangsvoraussetzung erwartet werden. Daß dies weithin nicht der Fall ist, muß mit Bedauern festgestellt werden.

Daraus wird die Forderung nach einer besseren Journalistenausbildung erhoben, die grundsätzlich auch erforderlich ist. Ob diese jedoch mit einer Fachhochschulausbildung erreicht wird, bleibt fraglich. Sicherlich wird man heute nicht mehr der Idee vom Journalistenberuf als "Begabungsberuf" anhängen ${ }^{30}$, aber in gewisser Weise ist er es dennoch. Gespür für Entwicklungen, Neugier, Kontaktbereitschaft etc. lassen sich kaum auf der Fachhochschule lernen. Im Blick auf die zur Erprobung anstehenden Ausbildungsmodelle (etwa in Dortmund) muß man mit einem abschließenden Urteil abwarten. Allerdings wird der offene Zugang zum Journalistenberuf auch weiterhin mit Nachdruck gegen alle Verschulungstendenzen verteidigt werden müssen ${ }^{37}$.

Wichtiger noch als neue Ausbildungsformen ist die Abschaffung des „Allround-Redakteurs" im Lokalen. Die zunehmend komplexer werdenden Lebensbezüge im kommunalen Raum erfordern den lokalen Fachredakteur, zumindest aber den Journalisten, der einige wenige Gebiete bearbeitet. Dies darf aber nicht so verstanden werden, daß sich eine Lokalredaktion künftig nur noch aus Spezialisten für Teilbereiche zusammensetzt, vielmehr so, daß nach einer allgemeinen Ausbildung für alle lokalen Bereiche eine Spezialisierung ermöglicht wird. Z. B. sind auch in einer wenig gegliederten Lokalredaktion folgende Ressorts möglich: Kommunalpolitik/Wirtschaft - Kultur/ Schule/Erwachsenenbildung - Soziales/Jugend/Gesundheit/Alte Menschen - Polizei/ Offentliche Sicherheit. Schwierig freilich dürfte eine solche Spezialisierung in jenen Lokalredaktionen fallen, die nur mit zwei oder drei Redakteuren besetzt sind (und das sind nicht wenige).

Wichtiger noch ist die Zielsetzung, die der Lokalzeitung gegeben wird ${ }^{38}$. Unbestritten dürfte sein, daß die Lokalzeitung als Informationsorgan der Honoratioren ${ }^{39}$ keine Zukunft mehr hat. Sie muß - wo dies nicht bereits geschehen ist - zum auditorium maximum werden, zu einem Markt für all die Menschen im überschaubaren 
Lebensraum, die etwas zu sagen haben (nicht nur im Sinn von Macht-haben, sondern vor allem im Sinn von Artikulation berechtigter Interessen, Wünsche etc. ${ }^{48}$ Darüber hinaus kommt der Lokalzeitung die Funktion zu, gesellschaftliche Funktion und Bedeutung im lokalen Bereich von überregionalen Ereignissen und Gesetzen aufzuzeigen, um so dem Bürger die Möglichkeit zu geben, sich aktiv am gesellschaftlichen und politischen Leben seines Lebensraumes zu beteiligen ${ }^{41}$. Nicht zuletzt muß die Lokalzeitung Sprachrohr für die Gruppen sein, die nicht über eine Lobby verfügen (Gastarbeiter, Behinderte, Kinder, Strafgefangene etc.).

Sollen diese Forderungen an eine moderne Lokalzeitung in die Tat umgesetzt werden, so setzt dies Lokaljournalisten voraus, die in der Lage sind, die sich vollziehenden gesellschaftlichen Prozesse auf der lokalen Ebene rechtzeitig zu erkennen. Darüber hinaus muß vom Lokaljournalisten ein ethisches Handlungsmodell erwartet werden, das sich nicht in einem oberflächlichen Pluralismus (Jedem das Seine) erschöpft, sondern in der Verantwortung für den Menschen verankert ist: Verantwortung für die Menschen, über die und für die berichtet wird.

An diesem Punkt freilich beginnt die Auseinandersetzung mit voller Schärfe: Wer bestimmt, was Verantwortung ist? Verantwortung an sich ist eine ebenso leere Formel wie leere Hülse, in die man zur Not jede Ideologie stecken kann. Außerdem wird es auch kaum möglich sein, eine Redaktionsgemeinschaft auf eine einheitliche ethische Norm festzulegen. Wäre dies überhaupt wünschenswert? Ein solcher Versuch, wollte man ihn unternehmen, hätte zweifellos im günstigsten Fall zahlreiche formale Lippenbekenntnisse zur Folge, die für die Tagespraxis letztlich nichts einbringen. Die ohnehin feststellbare Verschwommenheit ethischer Begründung journalistischen Handelns würde weiter zunehmen.

Was erreicht werden muß, ist (in knappen Zügen) dies: Auf der Grundlage einer Funktionsbestimmung für die Lokalzeitung ${ }^{42}$ ist ein Minimalkonsens für alle Redakteure festzulegen: Einhaltung der publizistischen Grundsätze ${ }^{43}$, Bemühen um sachliche Richtigkeit auch unter schwierigen alltäglichen Arbeitsbedingungen (was leider nicht immer selbstverständlich ist), Toleranz gegenüber Andersdenkenden, Förderung der Kommunikation unter den Menschen, Einsatz für den betreffenden lokalen Kommunikationsraum (ohne deshalb gleich Lokalpatriot zu werden), Förderung der wichtigen gesellschaftlichen Zeitfragen ${ }^{44}$, der Demokratie (ein Journalist sollte nicht nur Artikel 5 des Grundgesetzes - Meinungs- und Pressefreiheit - im Kopf haben, sondern vor allem auch Artikel 1 - Recht auf Würde eines jeden Menschen) und der sozialen Fortentwicklung (die vor allem für den Lokaljournalisten großer Ballungsräume immer wichtiger wird ${ }^{45}$ ).

Dieser "Minimalkonsens" bewahrt den Lokaljournalisten auch vor der Anfechtung, seine (angebliche) Macht auszuspielen, die ihm, nicht aber der Gesellschaft dient. Er bewahrt ihn auch davor, sich zum "Lehrer" aufzuspielen, was Arnold Gehlen beim 7. Salzburger Humanismusgespräch 1974 mit Recht als „störend“ bezeichnete, nämlic, „daß die Publizistik gegenüber den Massen eine penetrant pädagogische Haltung einnimmt, die in das Pflegerische übergeht, so daß ein lazaretthafter Zug die Publizistik" durchzieht ${ }^{48}$.

Auf diesem "Minimalkonsens" aufbauend - der freilich selbst eine ethische Grundentscheidung bereits voraussetzt - muß der Journalist seine Handlungsethik entfalten, die ihn in die Lage versetzt, unabhängig von Interessengruppen ${ }^{47}$ seinen Dienst für die Gesellschaft zu leisten. Dafür kommt er ohne eine fest verankerte Weltan- 
schauung nicht aus, sei sie nun christlich oder humanistisch begründet. Sie allein ermöglicht dem Journalisten die Prüfung und Unterscheidung der vielfältigen Zeitgeister und eben auch die innere Unabhängigkeit, ohne die ein der öffentlichen Verantwortung gerechtwerdender Journalismus nicht möglich ist. Wohl möglich - und notwendig - ist innerhalb der jeweiligen Redaktion die Pluralität verschiedener Weltanschauungen, die in der gemeinsamen Verantwortung für die Gesellschaft ihren gemeinsamen Nenner findet.

\section{Keine Angst vor der Partnerschaft}

Die Kirche besitzt eine große Chance, den Dialog mit den Massenmedien aufzunehmen, die selbst offensichtlich kaum in der Lage sind, einen eigenen überzeugenden Beitrag zur Ethik der Kommunikation und damit zur Verantwortung der Journalisten zu leisten. Drei Beispiele sollen zeigen, daß der Journalist auf der Suche nach seiner Verantwortung von der eigenen Lexika-Literatur allein gelassen wird. Aber was für die - so unterschiedlichen - Nachschlagewerke gilt, trifft für die gesamte neuere Literatur über die Massenkommunikation zu, die sich vorwiegend mit Soziologie, Psychologie, Statistik, Medienkritik und Mediendidaktik linker Provenienz sowie empirischen Untersuchungen zu Einzelfragen auseinandersetzt.

Unter dem Stichwort Verantwortung finden sich im Fischer-Lexikon „Publizistik ${ }^{48}$ nur die Verweise auf den verantwortlichen Redakteur und die Verantwortungsordnung bei Pressedelikten. Das Stichwort Ethik verweist auf einen Satz in der Einleitung: „Das derzeitige geringe - oder jedenfalls äußerst partielle - Interesse für ethische Fragen hat in den letzten zwei Jahrzehnten keine neuen Arbeiten zum Thema Ethik des Journalismus entstehen lassen. Wir haben darum auf einen Artikel dazu verzichtet. ${ }^{\alpha 40}$

Das „dtv-Wörterbuch zur Publizistik “50 zeigt sich etwas auskunftsfreudiger, aber nicht weniger verlegen. Unter dem Stichwort "Verantwortlichkeit" heißt es u.a.: „Nach demokratischer Auffassung stehen Freiheit und Verantwortung in einem direkten Verhältnis zueinander. ${ }^{\text {"51 }}$ Immerhin wird dem Stichwort „Ethik" ein eigener Abschnitt gewidmet mit Sätzen wie: „Obwohl berufsethische Grundprinzipien Allgemeingut geworden sind, konnten sie bisher nicht verbindlich formuliert werden. ${ }^{\text {"52 }}$

Werfen wir noch einen Blick in das „Wörterbuch der sozialistischen Journalistik ${ }^{\star \star 63}$. Die Stichworte „Moral“, „Verantwortung", „Ethik" fehlen. Aber sie sind in anderen Einzelbeiträgen aufgenommen. So heißt es im Abschnitt "Journalist": „Für den sozialistischen Journalist ist sein Beruf zugleich gesellschaftliche Berufung. Er gestaltet sein Leben nach den Grundsätzen der sozialistischen Moral und Ethik. Sein Berufsethos gebietet ihm, stets wahrheitsgemäß, verantwortungsbewußt, prinzipienfest und parteilich $\mathrm{zu}$ informieren und $\mathrm{zu}$ argumentieren." ${ }^{\text {"54 }}$

Die Kommunikation aber kann, wie Karl Steinbuch richtig feststellt, „so wenig wie ein Schiff nach einer Orientierungsmarke segeln, die an den eigenen Mast genagelt ist ${ }^{\alpha 35}$. Bietet die Kirche eine solche Orientierungsmarke, der nach dem Vorwort zum "Dekret über die sozialen Kommunikationsmittel " des II. Vatikanischen Konzils aus dem Jahr 1963 „sehr wohl bekannt (ist), daß die publizistischen Mittel bei rechter Verwendung den Menschen wirksame Hilfe bieten, denn sie leisten einen wichtigen Beitrag zur Erholung und Geistesbildung und dienen auch der Ausbreitung und Festigung des Gottesreiches" ${ }^{66}$ ? Und die Vollversammlung des Weltrates der Kirchen stellte 
1968 in Uppsala in ihrem Dokument „Die Kirche und die Medien der Massenkommunikation" ${ }^{\text {57 }}$ fest, daß den Massenmedien eine wichtige Rolle zukommt „als Treffpunkt und Versammlungsort der technologischen Gesellschaft ${ }^{\text {«58 }}$.

Die Kirche hat beispielsweise die Chance, mit ihrer eigenen Publizistik "Leuchttürme ${ }^{\text {" }}$ zu setzen. Doch dazu merkt Robert Geisendörfer für die evangelische Seite an: „Die Neigung, Publizistik als Instrument zu verstehen, wächst, wenn auch nicht im selben Maße, wie dies für profane Publizistik zu beobachten ist. ${ }^{\text {"59 }}$ Deutlicher hat dies Midhael Schmolke mit seiner „Krücken-These“ für die katholische Seite gesagt: „Zeiten der Bedrängnis machen nämlich klar, daß die Kirche erst auf dem Wege zum Heil ist, und daß man Krücken braucht, wenn man gehen will. Endet die Zeit der Bedrängnis, so gilt den Krücken erneut das prinzipielle Mißtrauen. ${ }^{\text {“80 }}$

Doch, um Michael Schmolke abermals zu Wort kommen zu lassen: „Wenn die Kirche ihrem Auftrag treu bleiben und die Gesellschaft durchdringen will, kommt sie ohne ein rationales, mehr noch: ein gutes Verhältnis zu den Medien nicht mehr aus. ${ }^{\text {011 }}$ Es geht also um die Partnerschaft von Kirche und Massenmedien, vor der sich freilich gegenwärtig noch beide Seiten fürchten: die Kirche, weil ihnen die Massenmedien letztlich nicht geheuer sind, die Journalisten, weil sie überall einen Machtanspruch der Kirche vermuten, den sie sich oft genug von Seiten der Politiker oder Verbände gefallen lassen, gegen die Kirche aber reagieren sie höchst sensibel und allergisch. Deshalb muß die Kirche künftig ihren Dialog - der freilich erst in Gang kommen muß - mit den Medien sehr uneigennnützig führen.

Was seitens der Kirchen theoretisch erkannt und in drei bedeutenden Dokumenten zum Problem der Massenkommunikation erklärt worden ist - gemeint sind die bereits erwähnten Dokumente „Dekret über die sozialen Kommunikationsmittel“ und "Die Kirche und die Medien der Massenkommunikation" sowie die Stellungnahme des Zentralkomitees der Deutschen Katholiken zur medienpolitischen Diskussion vom Herbst $1974^{62}$ - ist bislang kaum zu den Journalisten vorgedrungen und hat kaum einen Niederschlag in der Kirche am Ort gefunden. Der Eindruck drängt sich auf, daß die Kirchen bislang die Chance verkannt haben, die diese Dokumente bieten, und $\mathrm{da} ß$ es an der Fähigkeit fehlt, sie mit den notwendigen Konkretionen für den Journalisten zu versehen. Deshalb soll hier in aller Kürze eine Darstellung der drei Dokumente erfolgen, soweit sie für das zur Diskussion stehende Thema etwas aussagen.

Wenden wir uns zunächst dem vatikanischen Dekret $\mathrm{zu}^{0 \mathbf{2 a}}$, das feststellt, daß es „vor allem" die Aufgabe der Laien ist, "die publizistischen Mittel mit dem Geist der

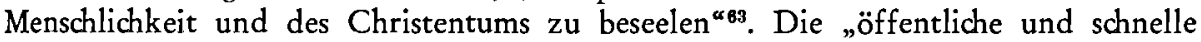
Berichterstattung" wird als "wirksamer Beitrag zum Gemeinwohl und zum Ausbau der bürgerlichen Gemeinschaft " angesehen ${ }^{84}$.

Der Journalist hat beim "Sammeln und Verbreiten von Nachrichten" ethische Grundsätze und die Würde des Menschen zu achten ${ }^{6}$. Deutlich wird hinzugefügt, daß „nicht alles Wissen Nutzen bringt" und unter Hinweis auf Paulus daran erinnert, daß allein die Liebe aufbaut ${ }^{65 \mathrm{a}}$. Das II. Vatikanische Konzil appelliert an die "besondere Verantwortung “ der Journalisten, deren "Information und Anregung dem Menschen Segen oder Fluch bringen (kann)" ${ }^{\text {"to }}$.

Ein solches Dekret muß zwangsläufig allgemein formuliert sein, es verweist aber im 2. Kapitel ausdrücklich auf die Aufgabe der Priester und Laien, sich verstärkt der Probleme der Massenkommunikation anzunehmen, proklamiert den Tag der sozi- 
alen Kommunikationsmittel ${ }^{67}$ und fügt hinzu: „Es ist der Kirche unwürdig, wenn ihre Glieder untätig zusehen müssen, wenn das Wort des Heils gefesselt und gehindert wird durch technische Unzulänglichkeit und ungenügende Mittel.“ब8

„Die Medien können das menschliche Wachstum hemmen", stellt das Mediendokument des Weltkirchenrates fest, vor allem dann, ,wenn ihr Hauptinteresse auf kommerzicllen Gewinn gerichtet ist ${ }^{\text {“8g. }}$. Die Kirche wird aufgefordert, sich nicht vor den Medien "als mächtiges Stimulans für das Mündigwerden des Menschen" zu fürchten und fährt dann fort: „Beängstigend ist jedoch die Tatsache, daß so wenige bereit sind, die Macht der Medien zu sehen und für ihre richtige Anwendung zu kämpfen. Das moderne Leben verlangt einen Preis für die Freiheit: die Bereitschaft, verantwortungsbewußt und diszipliniert für die neugewonnene Freiheit des Menschen zu handeln. ${ }^{470}$

$\mathrm{Zu}$ wenig öffentliche Beachtung hat bislang auch das Medienpapier des Zentralkomitees der deutschen Katholiken gefunden, das sich vorwiegend mit den medienpolitischen Vorstellungen der Parteien auseinandersetzt, aber bemerkenswerte Aussagen zur Situation der Publizistik macht. Das Zentralkomitee fordert "mehr Achtung vor dem Informationsanspruch des Bürgers"11, eine "Offenhaltung des Meinungskampfes ${ }^{\alpha 72}$, setzt sich für verschärfte Wettbewerbsregeln ein, um die Zeitungsvielfalt zu erhalten ${ }^{78}$ und für ein breites inhaltliches Spektrum bei Lokalmonopolen ${ }^{74}$.

Zur Berufsethik der Journalisten wird erklärt, daß sich dazu die Parteien bislang weitgehend ausgeschwiegen haben. Der Journalist wird vom Zentralkomitee aufgefordert, die Information nicht ,zur polit-pädagogischen Waffe umzufunktionieren ${ }^{\alpha 76}$ und fährt fort: „Das oberste Gesetz des Journalismus ist auch hier das Maß seines vermittelnden, öffentlichen Dienstes an der Wahrheit und der Information. ${ }^{{ }^{776}}$

Die Publizistik ist auf Theologie angewiesen, ,um ihre Praxis zu reflektieren und zu legitimieren "77 und die Kirche wird von den bedrängten kommunikationspolitischen Fragen herausgefordert, die nicht dadurch gelöst werden, „daß man sagt, wer sie lösen müßte. Denn was ist, wenn er sie nicht löst? ${ }^{\alpha 78}$

Der Journalist als „Arbeiter der Offentlichkeit ${ }^{\alpha 79}$ sieht sich heute vor Probleme gestellt, die ohne eine verantwortliche Ethik sehr bald einen großen gesellschaftlichen Schaden anrichten können.

In einer Partnerschaft zwischen Kirche und Massenmedien - die auf gegenseitiges Hören, Verstehen, Achten, Tolerieren und Helfen gegründet sein muß - ist die Kirche am Ort in folgenden Punkten herausgefordert:

1. Die Vielfalt der Presse, besonders im regionalen und lokalen Bereich ist gefährdet. Die Kirche muß darauf drängen, die Verantwortung der Verleger und Politiker so zu schärfen, daß die Erhaltung der Pressevielfalt mit allen möglichen Mitteln (notfalls mit öffentlichen Subventionen) gewährleistet wird. Denn im regionalen Bereich hat der Bürger mit nur einer Zeitung keine Chance mehr, sich anderen $\mathrm{Me}$ dien zuzuwenden ${ }^{80}$. Dies aber ist aufgrund der wachsenden Bedeutung der lokalen Kommunikation unbedingt notwendig.

2. Dort, wo inzwischen nur noch eine Lokalzeitung existiert - die wie Unkraut aus dem Boden schießenden Anzeigenblätter bilden kein Gegengewicht, sie gefährden höchstens die vorhandenen Lokalzeitungen durch den Entzug von Anzeigen -, muß die Kirche mit dazu beitragen, daß alle relevanten gesellschaftlichen Gruppen in der Monopolzeitung zu Wort kommen. Bei grober Verletzung der Meinungsvielfalt hat die Kirche laut und vernehmlich ihre Stimme zu erheben. 
3. Insgesamt sollte sich die Kirche verpflichtet fühlen, sich für eine möglichst große Zahl von Zeitungen einzusetzen, denn diese Mittel der Massenkommunikation begünstigen stets „liberale Tendenzen“, während die elektronischen Medien mehr „kollektivische Tendenzen" fördern ${ }^{81}$.

4. Der Kirche am Ort kommt ferner die Aufgabe zu, die lokalen Massenmedien stets daran zu erinnern, daß sie eine öffentliche Aufgabe zu erfüllen haben und im Dienst für den Menschen stehen, weil sie „öffentlicher Austragungsort für das Zeitgespräch der Gesellschaft" sind ${ }^{82}$. Das schließt die Mahnung an die Journalisten ein, ihre Macht nicht für bestimmte Ideologien oder eigennützige Zwecke zu mißbrauchen.

5. Der Einfluß der Massenmedien wächst. Die Zeit, die der Bürger heute für die Lektüre der Zeitungen, das Hören von Rundfunk und das Fernsehen aufwendet, steigt weiter $\mathrm{an}^{83}$. Z war ist die Wirkungsforschung noch nicht weit fortgeschritten ${ }^{84}$, doch ist davon auszugehen, daß langfristige Medienwirkungen nicht unterschätzt werden dürfen. Dadurch wächst die Verantwortung des Journalisten zusätzlich.

6. Offentliche und veröffentlichte Meinung dürfen nicht in einen unüberbrückbaren Gegensatz zueinander geraten ${ }^{85}$. Darauf wird eine verantwortliche Kirche hinweisen müssen.

7. Die Kirche kann aus ihrem Wissen und ihrem Eintreten für die Randgruppen der Gesellschaft die Medien auf diese Probleme aufmerksam machen und diese um eine stärkere Berücksichtigung bitten: Gastarbeiter, Obdachlose, Strafgefangene, mißhandelte Kinder etc.

8. Die Gefahr droht, daß die Medien immer mehr der Sensationsberichterstattung erliegen und dem sogenannten human touch einen ungebührend breiten Raum einräumen. Dabei werden nicht nur wichtige Nachrichten für den lokalen Kommunikationsraum verdrängt ${ }^{86}$, sondern es wird auch wenig Rücksicht auf die Betroffenen genommen (z. B. in der Polizei- und Gerichtsberichterstattung) ${ }^{87}$. Hier wird die Kirche das Augenmerk auf die menschlichen Maßstäbe lenken müssen.

9. Die oft harten Arbeitsbedingungen der Lokaljournalisten sind den Kirchen am Ort, die oft nur zu gern den Lokalteil kritisieren, meist aus recht eigennützigen Erwägungen heraus, nicht bekannt. Eine bessere Information ist dringend geboten, wodurch manches Vorurteil abgebaut werden kann.

10. Die Kirche hat auf der Seite der Journalisten zu stehen, die sich redlich bemühen, Mißstände im betreffenden Lebensraum aufzudecken und dadurch sich dem mächtigen Druck der Interessengruppen ausgesetzt sehen.

11. Uneigennützig sollte sich die Kirche allen Journalisten öffnen - und nicht nur denen, die Christen sind oder die man dafür hält.

12. Die Kirche sollte die Journalisten ermuntern, ihre Möglichkeiten zur besseren Kommunikation unter den Menschen auch zu nutzen, um so Lebensqualität und sozialen Wandel zu fördern.

Diese Aufgabe kann die Kirche freilich nur dann in Angriff nehmen, wenn sie ihre traditionelle Skepsis gegenüber den Journalisten aufgibt und endlich ihre Arbeit so würdigt, wie diese es redlicherweise verdient hat.

Der Göttinger Theologieprofessor Wolfgang Trillhaas hat kürzlich unter der Uberschrift "Die zehnte Muse" dem Journalisten ein Essay gewidmet, das nicht nur wegen seiner Treffsicherheit, sondern vor allem wegen seines tiefen Verständnisses für die Arbeit des Journalisten Beachtung verdient ${ }^{88}$. 
„Ergriffenheit und Distanznahme“ mache den Journalisten aus, dessen Aufgabe es sei, "unsere Zeit einzuatmen ${ }^{\text {c80 }}$. Ihm stehe es zu, die spitze Feder zu benutzen und „das Kind beim Namen zu nennen". Trillhaas weiter: „Es ist keine Verletzung journalistischer Leidenschaft, wenn man die Tränen der Traurigkeit nicht der Offentlichkeit preisgibt und wenn man über das Geheimnis des Sterbens den Mantel der Diskretion breitet. Ergriffenheit bedeutet für den Journalisten, daß die Maßstäbe des Menschlichen auch für ihn gelten. ${ }^{\alpha \theta 0}$

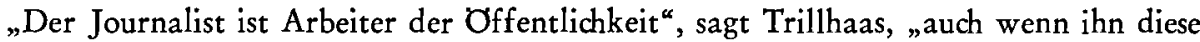
Offentlichkeit isolieren möchte. Er ist der Anwalt dessen, woran selbst Fachleute nicht denken, des Bewußtseins der Zeit. Und in alledem ist der Journalist verpflichtet, ein überlegener Mensch zu sein. ${ }^{{ }^{81}}$

\section{Das Gespräch sucben}

Die Freiheit der Lokalpresse (und nicht nur ihre) ist neben den Problemen, die sich aus der Pressekonzentration ergeben, davon abhängig, wieviel kleine Freiheiten der Journalist täglich verteidigt und erobert. Nicht Erzieher seiner Leser ist der Journalist, sondern Sachwalter der Informationen, die der Leser benötigt, um sich verantwortungsbewußt am gesellschaftlichen Leben zu beteiligen und sich in das soziale System seiner Umwelt integrieren zu können.

Um dies zu erreichen, muß der Journalist möglichst viel aus seiner „Umgebung“ herausgehen und das Gespräch mit Menschen suchen. Zugleich aber müssen die Menschen, die um das Problem der Verantwortung wissen, auf die Journalisten zugehen und gemeinsam immer wieder neu die schmale Fahrrinne praktischen ethischen Handelns ausloten.

$\mathrm{Daß}$ in diesem Gespräch der Kirche als Sachwalterin anthropologischen Wissens, über das keine andere Institution soviel weiß, eine besondere Rolle zukommt, versteht sich eigentlich von selbst und ist unter IV. aufgezeigt. Doch dauern Mißverständnisse und Vorurteile auf beiden Seiten weiter an. In der Uppsala-Erklärung des Weltkirchenrates über die Massenmedien ist dies in aller Ehrlichkeit ausgesprochen: „Die Welt der Massenkommunikationsmittel ist so neu und oft so verwirrend für die Kirche. ${ }^{{ }{ }^{2} 2}$ Hier liegen bislang ungenutzte Chancen, die für Journalisten und Kirche endlich genutzt werden müssen.

Für die Praxis bieten sich u. a. folgende Vorschläge an, ohne der Phantasie eine Grenze setzen zu wollen:

1. Zum Welttag der sozialen Kommunikationsmittel lädt die Kirche am Ort alle im entsprechenden Verbreitungsgebiet arbeitenden Journalisten zu einem Empfang ein, bei dem über gemeinsam interessierende Fragen gesprochen werden kann. Dazu bietet sich an, daß sowohl ein Pfarrer als auch ein Journalist zu einem bestimmten Thema ein Referat hält. Eine solche Veranstaltung eignet sich auch als eine ökumenische, denn die anstehenden Probleme gelten für alle Konfessionen. Und warum sollte in der Messe des betreffenden Sonntags nicht einmal ein Journalist eine Ansprache halten?

2. Der persönliche Kontakt zwischen örtlicher Kirchenleitung und den Lokalredaktionen ist zu intensivieren - durch Besuche in den Redaktionen seitens der Pfarrer, durch Einladung zu einem Gesprächsabend an Journalisten. 
3. Die kirchlichen Bildungswerke müssen mehr Themen aus dem Problemfeld der Massenkommunikation auf ihr Veranstaltungsprogramm setzen.

4. Die Kirche kann die Begegnung von Journalisten und Lesern fördern durch gemeinsame Vortragsabende.

Dies kann dazu beitragen, jenes Ziel zu erreichen, das im „mißachteten Leser" so formuliert ist: „Zuerst und in ihrer eigentlichen Berufsrolle haben Journalisten und Verleger ehrliche Makler, Spezialisten zur Berreuung des geistigen Austauschs in der Gesellschaft zu sein; eben dann Gesprächsanwälte und dann erst Vertreter des eigenen Standpunkts, zuerst Gesprächsleiter und Moderatoren und dann gleichberechtigte Mitsprecher im demokratischen Meinungsbildungsprozeß ${ }^{\alpha 93}$

Auf diesem Weg liegen noch viele Steine, doch diese dürfen nicht davon abhalten, täglich neu einige davon aus dem Weg zu räumen. Freilich, auch dies sollte realistischer Weise nicht verschwiegen werden: Das „ethische Berufsmaximum der Journalisten wird von der Realität soweit entfernt bleiben wie die Zehn Gebote von der menschlichen Wirklichkeit ${ }^{\text {(94 }}$.

\section{Anmerkungen:}

1. Zur Pressekonzentration vgl. Hermann Meyn: Massenmedien in der Bundesrepublik Deutschland. Neu bearbeitete Auflage 1974, Reihe "Zu Politik und Zeitgeschichte" Bd. 24, Berlin 1974, S. 60 ff. sowie K. Rüdiger Durth: Praktische Zeitungslehre. Journalismus im Grundriß, München 1974, S. 12. Nach Angaben von "Die Zeitung " 3 . Jhg. (1975), Nr. 11, S. 8, wurden im 3. Quartal 1975 in der Bundesrepublik Deutschland 20280283 Tageszeitungen täglich verkauft.

2. Hansjürgen Koschwitz: Publizistik und politisches System. Die internationale Presse der Gegenwart und ihre Entwidklungstendenzen in unterschiedlichen Herrschaftsformen. Piper-Sozialwissenschaft, Bd. 25, München 1974, S. 224.

3. „Die Zeitung ${ }^{\star}$, 4. Jhg. (1976), Heft 1, S. 7: Das Internationale Presseinstitut Zürich (IPI) stellte in seinem Jahresbericht 1975 fest, daß es in mehr als der Hälfte der Welt keine freie Presse gibt. Die einzige wirkliche Gefahr in der Bundesrepublik Deutschland sei allein wirtschaftlicher Natur, nicht politischer.

4. Aus der Fülle der neueren Literatur sei hier nur hingewiesen auf Dieter Prokop (Hrsg.): Kritische Kommunikationsforschung, Reihe Hanser, Bd. 141, München 1973, und Michael Buselmeier (Hrsg.): Das glückliche Bewußtsein. Anleitungen zur materialistischen Medienkritik. Sammlung Luchterhand, Bd. 1002, Neuwied 1974.

5. Vgl. dazu „Der Journalist ${ }^{\alpha}$, Heft 11, 1975, S. 35.

6. Vgl. Hermann Meyn: Massenmedien in der Bundesrepublik Deutschland, a. a. O., S. $82 \mathrm{ff}$.

7. Ebd. S. $39 \mathrm{ff}$.

8. Ebd. S. $79 \mathrm{ff}$.

9. Journalismus und kommunale Offentlichkeit. Ein Modell zur Fortbildung von Lokaljournalisten. Hausmitteilungen der Bundeszentrale für politische Bildung - Journalisten Bonn 1973, Heft 1, S. 12.

10. Vgl. dazu K. Rüdiger Durth: Der Lokalredakteur. Wege zur bürgernahen Kommunikation. Journalismus und Praxis, Heft 2, Remagen-Rolandseck 1975.

11. "Die Zeitung“, 3. Jhg. (1975), Heft 12, S. 5.

12. Ebd.; Thomas Gruber u. a.: Berufsziel: Journalist. Vorstellungen, Einstellungen und Bedingungen beim Eintritt in den Beruf. Themaheft "Journalismus als Beruf“, „Publizistik ${ }^{\star}$ 19. Jhg. (Heft 3-4) / 20. Jhg. (Heft 1-2), 1974/75, S. 347.

13. Das liegt nicht zuletzt daran, daß die geltenden Tarifverträge noch Auflagenbeschränkungen kennen und so selbst bei Mantelzeitungen für das Gehalt die Auflage der jeweiligen Lokalausgabe zugrunde gelegt wird. 
14. Aufermann/Elitz: Bildungs- und medienpolitische Perspektiven der Journalistenausbildung, in: Jörg Aufermann / Ernst Elitz (Hrsg.): Ausbildungswege zum Journalismus. Bestandsaufnahmen, Kritik und Alternativen der Journalistenausbildung. Studienbücher zur Sozialwissenschaft, Bd. 18, Opladen 1975, S. 244: „Die Bekenntnisse zur Notwendigkeit journalistischer Fortbildung ... sind bislang unverbindliches Wortgeklingel."

15. K. Rüdiger Durth: Lokale Kommunikation der Kirchen. Der Pfarrer und die Zeitung. Journalismus und Praxis, Heft 3, Remagen-Rolandseck 1975, S. $24 \mathrm{ff.}$

16. Abgedruckt in Durth: Praktische Zeitungslehre, a. a. O., S. $68 \mathrm{f}$.

17. Ernst Wolf: Schöpferische Nachfolge, in: Spannungsfelder der Evangelischen Soziallehre. Hrsg. von F. Karrenberg und W. Schweitzer, Bd. 7 der Studien zur Evangelischen Sozialtheologie und Sozialethik, Hamburg 1960, S. 38.

18. Michael Schmolke: Kirche, Gesellschaft und publizistische Medien. 100 Jahre PaulinusDruckerei Trier. Abgedruckt in Beilage 45/1975 „Aus Politik und Zeitgeschichte ${ }^{\alpha}$ zur Wochenzeitung „Das Parlament", S. 4.

19. Wie jüngst Konrad Kraemer: Das Recht auf Information - und die Pflicht, sich zu informieren, in: Kirchenzeitung für das Erzbistum Köln, Nr. 22/1976, S. 32.

20. Hans Reinhard Rapp bezeichnet in seinem Aufsatz "Nachhilfe für theologisches Reden? Vom Nutzen der Informationstheorie“, „Evangelische Kommentare“, 6. Jhg. (1976) S. 133, die Kirchen als "Informationsgräber", aus denen nichts mehr nach außen dringt.

21. Robert Geisendörfer: Publizistik als Geschäft der Kirche, in: „medium“, 6. Jhg. (1976), H. 4, S. 12.

22. Manfred Rühl: Die Zeitungsredaktion als organisiertes soziales System, 1969, S. 175 f.

23. Der Druck von außen, der auch personelle Konsequenzen nach sich zieht, ist nach wie vor die große Ausnahme. Meist arrangiert man sich.

24. Hier funktioniert öffentlicher Protest in der Regel vorzüglich, was die Praxis etwa der politischen Journalisten in der Bundeshauptstadt Bonn zeigt.

25. Peter Glotz / Wolfgang R. Langenbucher: Der mißachtete Leser. Zur Kritik der deutschen Presse. Köln/Berlin 1969, S. 122.

26. Beispielsweise vorgetragen in: Horst Haenisch / Klaus Schröter: Zum politischen Potential der Lokalpresse, in: Ralf Zoll (Hrsg.): Manipulation der Meinungsbildung. Zum Problem hergestellter Offentlichkeit. Kritik Bd. 4, 2. Aufl., Opladen 1972, S. $253 \mathrm{ff}$.

27. Vgl. K. Rüdiger Durth: Der freie Mitarbeiter an Tageszeitungen. Ein Leitfaden. Journalismus und Praxis, Heft 1, Remagen-Rolandseck 1975, S. 12 ff.

28. Ulrich Saxer: Dysfunktionale Folgen unzulänglicher Journalistenaus- und -fortbildung, in: Journalismus als Beruf, Publizistik-Themaheft, a. a. O., S. 296, über die Folgen der Arbeit von Pressestellen: „Die beim Recherchieren und anderswo feststellbare Veränderung des journalistischen Aktivitätsrayons kann sehr leicht zum Funktionsschwund des Journalismus durchschlagen, wenn dieser den Leistungen der oft besser ausgebildeten Spezialisten des neuen zweiten Berufssystems nicht gewachsen ist. ${ }^{*}$

29. Was Ministerpräsident Heinz Kühn in einem Interview zur Journalistenausbildung mit Aufermann/Elitz sagt, abgedruckt im Sammelband "Ausbildungswege zum Journalismus" dieser beiden Autoren, a. a. O., gilt erst recht für die Lokalzeitungen: Betrachtet man die Ausbildung, so sind ,immer wieder erstaunliche Leistungen an journalistischer Qualität zu registrieren ${ }^{\alpha}$ (S. 174). Bei der Bewertung der einzelnen Lokalteile muß man die teilweise großen Unterschiede $z$ wischen Lokalteilen von Großstadt- und Landzeitungen berücksichtigen, die sich schon aus der gegliederten Redaktionsstruktur von Großstadtzeitungen ergeben. Damit soll nicht gesagt sein, daß alle ländlichen Lokalzeitungen schlechter gemacht sind.

30. Das läßt sich bereits an den Stellenanzeigen in "Der Journalist“ der letzten beiden Jahre ablesen. Diese Monatszeitschrift bildet das widhtigste Publikationsorgan für Stellengesude und -angebote. Die Zahl der Angebote hat rapide abgenommen. Dieser Befund deckt sich auch mit eigenen Erfahrungen aus den Gesprächen mit Redakteuren.

31. Die Schlagzeile der Mai-Ausgabe 1976 des Monatsorgans der Deutschen JournalistenUnion "die feder": „Rekordgewinne der deutschen Tageszeitungen. Im Krisenjahr 1975 rund 1 Milliarde DM Gewinn" übersieht, daß die Gewinne von Großverlagen nicht einfach im Durchschnitt auf alle Zeitungen übertragen werden können. Im Gegenteil, viele mittlere Zeitungen haben im wahrsten Sinne des Wortes um ihre Existenz zu kämpfen. 
Zum anderen war 1975 aufgrund der unsicheren Lage auf dem Zeitungsmarkt die Investitionstätigkeit überdurchschnittlich gering. Investitionen aber sind gerade im Drudkbereich notwendig.

Die Frage der Investitionen im Blick auf die Einführung von Bildschirmgeräten und Lichtsatz muß hier unberücksichtigt bleiben. Es sei dafür auf den ausgezeichneten Beitrag von Dietrich Ratzke in der „Frankfurter Allgemeinen Zeitung“ vom 29. Mai 1976 unter der Überschrift "Der Redakteur am Bildschirm. Die Elektronik verdrängt das gute alte Blei" verwiesen.

32. Zum vieldiskutierten Mainzer und Dortmunder Modell für Journalisten-Ausbildung: "Die Zeitung“, 4. Jhg. (1976), Nr. 3, S. 5.

33. Vgl. Hans Heinz Fabris: Rekrutierung und Ausbildung des journalistischen Nachwuchses in der Presse der BRD, in: Aufermann/Elitz: Ausbildungswege zum Journalismus, a. a. O., S. $14 \mathrm{ff}$.

34. U. a. auch Thomas Gruber u. a.: Berufsziel: Journalist, a. a. O., S. 355.

35. Dies wird bei der Diskussion um das Dortmunder und Mainzer Modell ebenfalls deutlich, siehe unter Anmerkung 32. Nützliche Hinweise finden sich dazu in Walther von La Roche: Einführung in den praktischen Journalismus, München 1975.

36. Fabris: Rekrutierung und Ausbildung, a. a. O., S. 21.

37. Dies hat u. a. mit Nachdruck die Herbstvollversammlung 1974 des Zentralkomitees der deutschen Katholiken in einer "Stellungnahme zur medienpolitischen Diskussion" gefordert, in: CS 8 (1975), S. 180.

38. Ausführliches dazu in K. R. Durth: Der Lokalredakteur, a. a. O., S. $19 \mathrm{ff}$.

39. Ebd. S. $23 \mathrm{ff}$.

40. Glotz/Langenbucher: Der mißachtete Leser, a. a. O., S. 122.

41. Siehe Anmerkung 38.

42. Durth: Der Lokalredakteur, a. a. O.

43. Siehe Anmerkung 16.

44. In seiner Rede zum 65jährigen Bestehen der Max-Planck-Gesellschaft im Juni 1976 hat Bundespräsident Walter Scheel mit Recht die Massenmedien aufgefordert, sidh mehr mit Problemen wie Umweltgefährdung, Zusammenleben in Ballungsräumen und Gesundheitsfragen zu beschäftigen. Süddeutsche Zeitung vom 26./27. Juni 1976.

45. Jürgen Tern: Der kritische Zeitungsleser. Bedk'sche Schwarze Reihe, Bd. 99, München 1973 , S. 65 f.

46. Arnold Gehlen: Die gewaltlose Lenkung. Zur Funktion der Massenmedien in der modernen Gesellschaft, in: Oskar Schatz (Hrsg.): Die elektronische Revolution. Wie gefährlich sind die Massenmedien?, Graz/Wien/Köln 1975, S. 64.

47. Hier müßte die Frage diskutiert werden, ob ein Journalist einer politischen Partei angehören sollte. Er ist Staatsbürger wie jeder andere auch, und es ist selbstverständlich, daß er über einen festen politischen Standort verfügt. Ist er Mitglied einer Partei, dann sollte er jedoch über soviel Souveränität verfügen, daß er seine Stellung nicht mißbraudit, in seiner Zeitung seine Partei zu fördern. Von einer aktiven politischen Betätigung ist abzuraten, um Interessenkollisionen zu vermeiden.

48. Fischer-Lexikon „Publizistik", hrsg. von Elisabeth Noelle-Neumann und Winfried Schulz, Bd. 9, 1971.

49. Ebd. S. 9.

50. dtv-Wörterbuch zur Publizistik, hrsg. von Kurt Koszyk und Karl H. Pruys, 1969.

51. Ebd. S. 364.

52. Ebd. S. $90 \mathrm{f}$.

53. Wörterbud der sozialistischen Journalistik, hrsg. von Emil Dusiska, Leipzig 1973 (als Manuskript gedrudkt).

54. Ebd. S. 116.

55. Karl Steinbuch: Kommunikation als technisches und gesellschaftliches Phänomen, in: Oskar Schatz, Die elektronische Revolution, a. a. O., S. 45.

56. Dekret über die sozialen Kommunikationsmittel „Inter Mirifica“. Konzilstexte-Deutsch, Heft 16, Trier 1966, S. 1. 
57. Die Kirche und die Medien der Massenkommunikation, in: „Missionierende Gemeinde", Heft 19 „Kirchliche Kontakte mit Publizisten“, Berlin 1969, S. 20 ff.

58. Ebd. S. 20.

59. Robert Geisendörfer: Publizistik als kirchliche Aufgabe. Skizze eines medienpolitischen Gesamtkonzepts, in: „Evangelische Kommentare“ 8 (1975), S. 480.

60. Michael Schmolke: Kirche, Gesellschaft und publizistische Medien, a. a. O., S. 8.

61. Ebd.

62. Abgedruckt in CS 8 (1975), S. 177-180. Die beachtenswerte Einleitung zu dieser Stellungnahme von Michael Albus findet sich im gleichen Heft, S. 169-176.

62a. Die Pastoralinstruktion "Communio et Progressio“ fügt in den hier zitierten Aussagen keine wesentlichen neuen Aspekte hinzu. Deshalb beschränke ich mich auf das Konzildekret, das $\mathrm{ja}$ in einer direkten zeitlichen und sachlichen Parallele zum Uppsala-Dokument steht.

63. A. a. O., S. 2.

64. Ebd. S. 3.

65. Ebd.

65a. Ebd.

66. Ebd. S. 5.

67. Ebd. S. $7 \mathrm{ff}$.

68. Ebd. S. 9.

69. A. a. O., S. 21.

70. Ebd. S. 26.

71. A. a. O., S. 177.

72. Ebd. S. 178.

73. Ebd. S. 179.

74. Ebd.

75. Ebd. S. 180.

76. Ebd. S. $180 \mathrm{f}$.

77. Robert Geisendörfer: Publizistik als kirchlidhe Aufgabe, a. a. O., S. 481.

78. Robert Geisendörfer: Publizistik als Geschäft der Kirche, a. a. O., S. 12.

79. Wolfgang Trillhaas: Die zehnte Muse, in: „Evangelische Kommentare ${ }^{c} 9$ (1976), S. 44.

80. Jürgen Hüther: Sozialisation durch Massenmedien. Ziele, Methoden, Ergebnisse einer medienbezogenen Jugendkunde, Opladen 1975, S. 69; Hans Edkehard Bahr: Verkündigung als Information, Konkretionen Bd. 1, Hamburg 1968, S. 139.

81. Karl Steinbuch: Kommunikation als technisches und gesellschaftliches Phänomen, a. a. O., S. 31 .

82. Hans Eckehard Bahr: Verkündigung als Information, a. a. O., S. 109.

83. Gertraude Steindl: Politisch Interessierte lesen' mehr, in: „Die Zeitung ${ }^{\alpha}$, 3. Jhg. (1975), Heft 9, S. 14: Die Zahl der Haushalte, die ein Fernsehen besaß, stieg von 1964 von 55 auf 95 in 1974 (Index: 100 Haushalte), die Zahl der Rundfunkgeräte im gleichen Zeitraum von 95 auf 96 und die der Zeitungen von 70 auf 78 . An einem durchschnittlichen Werktag wurden 197443 Prozent aller Haushalte von drei Medien erreicht, 40 Prozent von $z$ wei Medien. 97 Prozent von mindestens einem. Der Zeitaufwand für die Nutzung der Medien wuchs von 1964 bis 1974 um durchschnittlich 78 Minuten und betrug 1974 durchschnittlich dreieinhalb Stunden pro Tag (2 Stunden, 11 Minuten TV, 1 Stunde, 52 Minuten Hörfunk, 38 Minuten Zeitung). Gegenüber 1970 hat sich der Zeitaufwand der Kernleserschaft der Tageszeitung mit starkem politischen Interesse weiter erhöht und lag 1974 um durchschnittlich 10 Minuten höher. Die Nutzung des Lokalteils stieg im Zeitraum 1970 bis 1974 von 55 auf 61 Prozent, die des politischen Teils von 49 auf 54 Prozent.

84. Jürgen Hüther: Sozialisation durch Massenmedien, a. a. O., S. 67.

85. Karl Steinbuch: Kommunikation als technisches und gesellschaftliches Problem, a.a. O., S. 47: "Die Distanz zwischen öffentlicher und veröffentlichter Meinung ist wohl die schwerste Bedrohung unserer liberalen Lebensform." 
86. Hans Eckehard Bahr: Verkündigung als Information, a. a. O., S. 138: „Ein Ergebnis der Kommerzialisierung von Nachrichten ist die Herstellung einer Uberfülle von Informationen, deren Informationswert jedoch gänzlich fragwürdig ist." Mediendokument "Die Kirche und die Medien der Massenkonmunikation", a. a.O., S. 29: „Nachrichten und Informationen sollten ein genaues Bild des menschliden Lebens vermitteln und nidht nur das Bizarre und Sensationelle berichten."

87. Vgl. Kaarle Nordenstreng u. a.: Grundsätze der Nachrichtenvermittlung, in: Gerhard Maletzke (Hrsg.): Einführung in die Massenkommunikationsforschung, Berlin 1972, S. 109 ff.; Karl Steinbuch: Kurskorrektur, Stuttgart 1973, S. 89: „Wer fragt schon bei publizierten Ideen, ob sie intellektuellen oder moralischen Mindestanforderungen genügen?", und S. 104: „Die Lust am Skandal bringt den publizistischen Profit, nicht der banale Ernst."

88. Wolfgang Trillhass: Die zehnte Muse, a.a. O., S. $42 \mathrm{ff}$.

89. Ebd. S. 42.

90. Ebd. S. 43.

91. Ebd. S. 44.

92. A. a. O., S. 33.

93. P. Glotz / W. R. Langenbucher: Der mißachtete Leser, a. a. O., S. 29.

94. Karl-Hermann Flach, Macht und Elend der Presse, Mainz 1967, S. 189.

\section{S U M M A R Y}

The responsibility of the local journalist in his day to day work should be also considered by the Church. She has to see actually her role for people working in the mass media not to underline her power but as a service to people who carry special responsibility in society. "The Church has a great chance to take up dialogue with the mass media who hardly seem to be able themselves to contribute towards the ethics of communications and with that towards the responsibility of the journalists." Communications ("Publizistik") relies on theology, nin oder to reflect her practical performance and legitimize it". A number of conditions for partnership between Church and mass media are listed as well as proposals for practical co-operation. Obstacles in this relation one should attempt to remove day by day since - as Karl Hermann Flach said - the actual fact sometimes might be apart as much as the ten commandments from human reality.

\section{RÉS U M É}

L'auteur traite de la responsabilité originale du journaliste, telle qu'elle se présente à lui, dans le problème de la liberté de la presse, dans la vie quotidienne du rédacteur (local). L'Eglise a en même temps une fonction importante à remplier. Elle le peut plus elle apprend à comprendre qu'elle a une "responsabilité bien concrête à assumer vis-à-vis des mass media et de ses collaborateurs -assurément pas dans le sens d'une revendication de pouvoir secrête ou ouverte, mais en tant que service envers les hommes qui ressentent une responsabilité particulière dans la société actuelle“. "L'Eglise possède la grande chance de pouvoir ouvrir le dialogue avec les mass media qui sont eux-mêmes apparemment dans l'impossibilité de 
produire une participation propre et persuasive au sujet de l'éthique de la communication et par là au sujet de la responsabilité des journalistes". L'auteur proclame avec Robert Geisendörfer que la publicistique a besoin de la théologie "pour réflecter et légitimer sa pratique“. L'auteur plaide en vue d'une association entre l'Eglise et les mass media, il énumère une série de réclamations et offre aussi une série de propositions pour la pratique. Il est conscient de ce que plus d'une difficulté fait obstacle à une bonne association, mais elles devraient être abolies par un effort quotidien, même si idéal - comme Karl-Hermann Flach l'écrivait reste autant éloigné de la réalité que les dix commandements de la réalité humaine“.

\section{R E S U M E N}

El autor trata de la responsabilidad del periodista tal como se le plantea a diario al redactor (local) en relación con el problema de la libertad de prensa. En este contexto tiene la Iglesia una importante misión que cumplir. Tanto más, en la medida que comprenda que tiene „una responsabilidad muy concreta respecto de los medios de difusión y sus colaboradores - no en el sentido de reivindicar un poder velado ó público, sino como servicio a los hombres que tienen en la sociedad actual una responsabilidad especial“. La Iglesia posee „una gran oportunidad de iniciar el diálogo con los medios de difusión que apenas se encuentran en situación de prestar por sí mismos una aportación convincente a la ética de la comunicación social y, consiguientemente, a la responsabilidad del periodista ${ }^{\star}$. El publicismo depende de la teología, dice el autor, remedando a Robert Geisendörfer, „para reflectar y legitimizar su práctica". En orden a la colaboración entre Iglesia y medios de difusión, por la que aboga el autor, presenta una serie de urgencias y ofrece una serie de propuestas para la práctica. Es consciente de que existen aún una serie de obstáculos a la colaboración, que no obstante deben ser superados en la práctica diaria, incluso si el ideal - como escribe Karl Hermann Flach - "dista mucho de la realidad" al igual que "los diez mandamientos de la realidad humana ${ }^{\alpha}$. 